\title{
Calkin images of Fourier convolution operators with slowly oscillating symbols
}

\author{
C. A. Fernandes, A. Yu. Karlovich and Yu. I. Karlovich
}

\begin{abstract}
Let $\Phi$ be a $C^{*}$-subalgebra of $L^{\infty}(\mathbb{R})$ and $S O_{X(\mathbb{R})}^{\diamond}$ be the Banach algebra of slowly oscillating Fourier multipliers on a Banach function space $X(\mathbb{R})$. We show that the intersection of the Calkin image of the algebra generated by the operators of multiplication $a I$ by functions $a \in \Phi$ and the Calkin image of the algebra generated by the Fourier convolution operators $W^{0}(b)$ with symbols in $S O_{X(\mathbb{R})}^{\diamond}$ coincides with the Calkin image of the algebra generated by the operators of multiplication by constants.
\end{abstract}

Mathematics Subject Classification (2010). Primary 47G10, Secondary 42A 45, 46E30.

Keywords. Fourier convolution operator, Fourier multiplier, multiplication operator, slowly oscillating function, Calkin algebra, Calkin image.

\section{Introduction}

Let $\mathcal{F}: L^{2}(\mathbb{R}) \rightarrow L^{2}(\mathbb{R})$ denote the Fourier transform

$$
(\mathcal{F} f)(x):=\widehat{f}(x):=\int_{\mathbb{R}} f(t) e^{i t x} d t, \quad x \in \mathbb{R},
$$

and let $\mathcal{F}^{-1}: L^{2}(\mathbb{R}) \rightarrow L^{2}(\mathbb{R})$ be the inverse of $\mathcal{F}$,

$$
\left(\mathcal{F}^{-1} g\right)(t)=\frac{1}{2 \pi} \int_{\mathbb{R}} g(x) e^{-i t x} d x, \quad t \in \mathbb{R} .
$$

It is well known that the Fourier convolution operator

$$
W^{0}(a):=\mathcal{F}^{-1} a \mathcal{F}
$$

is bounded on the space $L^{2}(\mathbb{R})$ for every $a \in L^{\infty}(\mathbb{R})$.

This work was partially supported by the Fundação para a Ciência e a Tecnologia (Portuguese Foundation for Science and Technology) through the project UID/MAT/00297/2019 (Centro de Matemática e Aplicações). The third author was also supported by the SEPCONACYT Project A1-S-8793 (México). 
Let $X(\mathbb{R})$ be a Banach function space and $X^{\prime}(\mathbb{R})$ be its associate space. Their technical definitions are postponed to Section 2.1 The class of Banach function spaces is very large. It includes Lebesgue, Orlicz, Lorentz spaces, variable Lebesgue spaces and their weighted analogues (see, e.g., [4, 6]). Let $\mathcal{B}(X(\mathbb{R}))$ denote the Banach algebra of all bounded linear operators acting on $X(\mathbb{R})$, let $\mathcal{K}(X(\mathbb{R}))$ be the closed two-sided ideal of all compact operators in $\mathcal{B}(X(\mathbb{R}))$, and let $\mathcal{B}^{\pi}(X(\mathbb{R}))=\mathcal{B}(X(\mathbb{R})) / \mathcal{K}(X(\mathbb{R}))$ be the Calkin algebra of the cosets $A^{\pi}:=A+\mathcal{K}(X(\mathbb{R}))$, where $A \in \mathcal{B}(X(\mathbb{R}))$.

If $X(\mathbb{R})$ is separable, then $L^{2}(\mathbb{R}) \cap X(\mathbb{R})$ is dense in $X(\mathbb{R})$ (see Lemma 2.1 below). A function $a \in L^{\infty}(\mathbb{R})$ is called a Fourier multiplier on $X(\mathbb{R})$ if the convolution operator $W^{0}(a)$ defined by (1.1) maps $L^{2}(\mathbb{R}) \cap X(\mathbb{R})$ into $X(\mathbb{R})$ and extends to a bounded linear operator on $X(\mathbb{R})$. The function $a$ is called the symbol of the Fourier convolution operator $W^{0}(a)$. The set $\mathcal{M}_{X(\mathbb{R})}$ of all Fourier multipliers on $X(\mathbb{R})$ is a unital normed algebra under pointwise operations and the norm

$$
\|a\|_{\mathcal{M}_{X(\mathbb{R})}}:=\left\|W^{0}(a)\right\|_{\mathcal{B}(X(\mathbb{R}))} .
$$

For a unital $C^{*}$-subalgebra $\Phi$ of the algebra $L^{\infty}(\mathbb{R})$, we consider the quotient algebra $\mathcal{M O}^{\pi}(\Phi)$ consisting of the cosets

$$
[a I]^{\pi}:=a I+\mathcal{K}(X(\mathbb{R}))
$$

of multiplication operators by functions in $\Phi$ :

$$
\mathcal{M O}^{\pi}(\Phi):=\left\{[a I]^{\pi}: a \in \Phi\right\}=\{a I+\mathcal{K}(X(\mathbb{R})): a \in \Phi\} .
$$

For a unital Banach subalgebra $\Psi$ of the algebra $\mathcal{M}_{X(\mathbb{R})}$, we also consider the quotient algebra $\mathcal{C O}^{\pi}(\Psi)$ consisting of the cosets

$$
\left[W^{0}(b)\right]^{\pi}:=W^{0}(b)+\mathcal{K}(X(\mathbb{R}))
$$

of convolution operators with symbols in the algebra $\Psi$ :

$$
\mathcal{C O}^{\pi}(\Psi):=\left\{\left[W^{0}(b)\right]^{\pi}: b \in \Psi\right\}=\left\{W^{0}(b)+\mathcal{K}(X(\mathbb{R})): b \in \Psi\right\} .
$$

It is easy to see that $\mathcal{M O}^{\pi}(\Phi)$ and $\mathcal{C O}^{\pi}(\Psi)$ are commutative unital Banach subalgebras of the Calkin algebra $\mathcal{B}^{\pi}(X(\mathbb{R}))$. It is natural to refer to the algebras $\mathcal{M O}^{\pi}(\Phi)$ and $\mathcal{C O}^{\pi}(\Psi)$ as the Calkin images of the algebras $\mathcal{M O}(\Phi)=\{a I: a \in \Phi\} \subset \mathcal{B}(X(\mathbb{R})), \mathcal{C O}(\Psi)=\left\{W^{0}(b): b \in \Psi\right\} \subset \mathcal{B}(X(\mathbb{R}))$, respectively. The algebras $\mathcal{M O}(\Phi)$ and $\mathcal{C O}(\Psi)$ are building blocks of the algebra of convolution type operators

$$
\mathcal{A}(\Phi, \Psi ; X(\mathbb{R}))=\operatorname{alg}_{\mathcal{B}(X(\mathbb{R}))}\left\{a I, W^{0}(b): a \in \Phi, b \in \Psi\right\},
$$

the smallest closed subalgebra of $\mathcal{B}(X(\mathbb{R}))$ that contains the algebras $\mathcal{M O}(\Phi)$ and $\mathcal{C O}(\Psi)$.

Let $S O^{\diamond}$ be the $C^{*}$-algebra of slowly oscillating functions and $S O_{X(\mathbb{R})}^{\diamond}$ be the Banach algebra of all slowly oscillating Fourier multipliers on the space $X(\mathbb{R})$, which are defined below in Sections 2.5 2.7. The third author proved 
in [23. Lemma 4.3] in the case of Lebesgue spaces $L^{p}(\mathbb{R}, w), 1<p<\infty$, with Muckenhoupt weights $w \in A_{p}(\mathbb{R})$ that

$$
\mathcal{M O}^{\pi}\left(S O^{\diamond}\right) \cap \mathcal{C O}^{\pi}\left(S O_{L^{p}(\mathbb{R}, w)}^{\diamond}\right)=\mathcal{M O}^{\pi}(\mathbb{C})
$$

where

$$
\mathcal{M O}^{\pi}(\mathbb{C}):=\left\{[c I]^{\pi}: c \in \mathbb{C}\right\} .
$$

This result allowed him to describe the maximal ideal space of the commutative Banach algebra

$$
\mathcal{A}^{\pi}\left(S O^{\diamond}, S O_{L^{p}(\mathbb{R}, w)}^{\diamond} ; L^{p}(\mathbb{R}, w)\right)=\mathcal{A}\left(S O^{\diamond}, S O_{L^{p}(\mathbb{R}, w)}^{\diamond} ; L^{p}(\mathbb{R}, w)\right) / \mathcal{K}\left(L^{p}(\mathbb{R}, w)\right)
$$

(see [23, Theorem 3.1]). In turn, this description plays a crucial role in the study of the Fredholmness of operators in more general algebras of convolution type operators with piecewise slowly oscillating data on weighted Lebesgue space $L^{p}(\mathbb{R}, w)$ (see [23, 24, 25]).

Recall that the (non-centered) Hardy-Littlewood maximal function $\mathcal{M f}$ of a function $f \in L_{\text {loc }}^{1}(\mathbb{R})$ is defined by

$$
(\mathcal{M} f)(x):=\sup _{I \ni x} \frac{1}{|I|} \int_{I}|f(y)| d y,
$$

where the supremum is taken over all intervals $I \subset \mathbb{R}$ of finite length containing $x$. The Hardy-Littlewood maximal operator $\mathcal{M}$ defined by the rule $f \mapsto \mathcal{M f}$ is a sublinear operator.

The aim of this paper is to extend (1.2) to the case of separable Banach function spaces such that the Hardy-Littlewood maximal operator $\mathcal{M}$ is bounded on $X(\mathbb{R})$ and on its associate space $X^{\prime}(\mathbb{R})$ and to the case of arbitrary algebras of functions $\Phi \subset L^{\infty}(\mathbb{R})$ in place of $S O^{\diamond}$.

The following statement extends [23, Lemma 4.3].

Theorem 1.1 (Main result). Let $X(\mathbb{R})$ be a separable Banach function space such that the Hardy-Littlewood maximal operator $\mathcal{M}$ is bounded on the space $X(\mathbb{R})$ and on its associate space $X^{\prime}(\mathbb{R})$. If $\Phi$ is a unital $C^{*}$-subalgebra of $L^{\infty}(\mathbb{R})$, then

$$
\mathcal{M O}^{\pi}(\Phi) \cap \mathcal{C O} \mathcal{O}^{\pi}\left(S O_{X(\mathbb{R})}^{\diamond}\right)=\mathcal{M O}^{\pi}(\mathbb{C})
$$

where $\mathcal{M O}^{\pi}(\mathbb{C})$ is defined by (1.3).

This result is one more step towards the study of Fredholm properties of convolution type operators with discontinuous data on Banach function spaces more general than weighted Lebesgue spaces initiated in the authors works [8, 9, 10.

One can expect, by analogy with the case of weighted Lebesgue spaces, that, for instance, $\mathcal{K}(X(\mathbb{R})) \subset \mathcal{A}\left(S O^{\diamond}, S O_{X(\mathbb{R})}^{\diamond} ; X(\mathbb{R})\right)$ and that the quotient algebra

$$
\mathcal{A}^{\pi}\left(S O^{\diamond}, S O_{X(\mathbb{R})}^{\diamond} ; X(\mathbb{R})\right)=\mathcal{A}\left(S O^{\diamond}, S O_{X(\mathbb{R})}^{\diamond} ; X(\mathbb{R})\right) / \mathcal{K}(X(\mathbb{R}))
$$

is commutative. It seems, however, that the proofs of both hypotheses will require tools, which are not available in the setting of general Banach function spaces. We plan to return to these questions in a forthcoming work, restricting 
ourselves to particular Banach function spaces, like rearrangement-invariant spaces with Muckenhoupt weights or variable Lebesgue spaces, where interpolation theorems are available.

The paper is organized as follows. In Section [2, we collect necessary facts on Banach function spaces and Fourier multipliers on them. Further, we recall the definition of the $C^{*}$-algebra $S O^{\diamond}$ of slowly oscillating functions and introduce the Banach algebra of slowly oscillating Fourier multipliers $S O_{X(\mathbb{R})}^{\diamond}$ on a Banach function spaces $X(\mathbb{R})$. In Section 3 , we discuss the structure of the maximal ideal spaces $M\left(S O^{\diamond}\right)$ and $M\left(S O_{X(\mathbb{R})}^{\diamond}\right)$ of the $C^{*}$ algebra $S O^{\diamond}$ of slowly oscillating functions and the Banach algebra $S O_{X(\mathbb{R})}^{\diamond}$ of slowly oscillating Fourier multipliers on a Banach function space $X(\mathbb{R})$. In particular, we show that the fibers $M_{t}\left(S O^{\diamond}\right)$ of $M\left(S O^{\diamond}\right)$ over the points $t \in \dot{\mathbb{R}}:=\mathbb{R} \cup\{\infty\}$ can be identified with the fibers $M_{t}\left(S O_{t}\right)$, where $S O_{t}$ is the $C^{*}$-algebra of all bounded continuous functions on $\dot{\mathbb{R}} \backslash\{t\}$ that slowly oscillate at the point $t$. An analogous result is also obtained for the fibers of the maximal ideal spaces of algebras of slowly oscillating Fourier multipliers on a Banach function space $X(\mathbb{R})$. In Section 4, we show that the maximal ideal spaces of the algebras $\mathcal{M} \mathcal{O}^{\pi}(\Phi)$ and $\mathcal{C O}^{\pi}(\Psi)$ are homeomorphic to the maximal ideal spaces of the algebras $\Phi$ and $\Psi$, respectively, where $\Phi$ is a unital $C^{*}$-subalgebra of $L^{\infty}(\mathbb{R})$ and $\Psi$ is a unital Banach subalgebra of $\mathcal{M}_{X(\mathbb{R})}$. In Section 5, we recall the definition of a limit operator (see [26] for a general theory of limit operators), as well as, a known fact about limit operators of compact operators acting on Banach function spaces. Further, we calculate the limit operators of the Fourier convolution operator $W^{0}(b)$ with a slowly oscillating symbol $b \in S O_{X(\mathbb{R})}^{\diamond}$. Finally, gathering the above mentioned results on limit operators, we prove Theorem 1.1.

\section{Preliminaries}

\subsection{Banach function spaces}

The set of all Lebesgue measurable complex-valued functions on $\mathbb{R}$ is denoted by $\mathfrak{M}(\mathbb{R})$. Let $\mathfrak{M}^{+}(\mathbb{R})$ be the subset of functions in $\mathfrak{M}(\mathbb{R})$ whose values lie in $[0, \infty]$. The Lebesgue measure of a measurable set $E \subset \mathbb{R}$ is denoted by $|E|$ and its characteristic function is denoted by $\chi_{E}$. Following [4, Chap. 1, Definition 1.1], a mapping $\rho: \mathfrak{M}^{+}(\mathbb{R}) \rightarrow[0, \infty]$ is called a Banach function norm if, for all functions $f, g, f_{n}(n \in \mathbb{N})$ in $\mathfrak{M}^{+}(\mathbb{R})$, for all constants $a \geq 0$, and for all measurable subsets $E$ of $\mathbb{R}$, the following properties hold:

$$
\begin{aligned}
& \rho(f)=0 \Leftrightarrow f=0 \text { a.e., } \quad \rho(a f)=a \rho(f), \quad \rho(f+g) \leq \rho(f)+\rho(g) \\
& 0 \leq g \leq f \text { a.e. } \Rightarrow \rho(g) \leq \rho(f) \quad \text { (the lattice property) } \\
& 0 \leq f_{n} \uparrow f \text { a.e. } \Rightarrow \rho\left(f_{n}\right) \uparrow \rho(f) \quad \text { (the Fatou property) } \\
& |E|<\infty \Rightarrow \rho\left(\chi_{E}\right)<\infty \\
& |E|<\infty \Rightarrow \int_{E} f(x) d x \leq C_{E} \rho(f)
\end{aligned}
$$


with $C_{E} \in(0, \infty)$ which may depend on $E$ and $\rho$ but is independent of $f$. When functions differing only on a set of measure zero are identified, the set $X(\mathbb{R})$ of all functions $f \in \mathfrak{M}(\mathbb{R})$ for which $\rho(|f|)<\infty$ is called a Banach function space. For each $f \in X(\mathbb{R})$, the norm of $f$ is defined by

$$
\|f\|_{X(\mathbb{R})}:=\rho(|f|) .
$$

Under the natural linear space operations and under this norm, the set $X(\mathbb{R})$ becomes a Banach space (see [4, Chap. 1, Theorems 1.4 and 1.6]). If $\rho$ is a Banach function norm, its associate norm $\rho^{\prime}$ is defined on $\mathfrak{M}^{+}(\mathbb{R})$ by

$$
\rho^{\prime}(g):=\sup \left\{\int_{\mathbb{R}} f(x) g(x) d x: f \in \mathfrak{M}^{+}(\mathbb{R}), \rho(f) \leq 1\right\}, \quad g \in \mathfrak{M}^{+}(\mathbb{R}) .
$$

It is a Banach function norm itself [4, Chap. 1, Theorem 2.2]. The Banach function space $X^{\prime}(\mathbb{R})$ determined by the Banach function norm $\rho^{\prime}$ is called the associate space (Köthe dual) of $X(\mathbb{R})$. The associate space $X^{\prime}(\mathbb{R})$ is naturally identified with a subspace of the (Banach) dual space $[X(\mathbb{R})]^{*}$.

\subsection{Density of nice functions in separable Banach function spaces}

As usual, let $C_{0}^{\infty}(\mathbb{R})$ denote the set of all infinitely differentiable functions with compact support.

Lemma 2.1 ([8, Lemma 2.1] and [22, Lemma 2.12(a)]). If $X(\mathbb{R})$ is a separable Banach function space, then the sets $C_{0}^{\infty}(\mathbb{R})$ and $L^{2}(\mathbb{R}) \cap X(\mathbb{R})$ are dense in the space $X(\mathbb{R})$.

Let $\mathcal{S}(\mathbb{R})$ be the Schwartz space of rapidly decreasing smooth functions and let $\mathcal{S}_{0}(\mathbb{R})$ denote the set of functions $f \in \mathcal{S}(\mathbb{R})$ such that their Fourier transforms $\mathcal{F} f$ have compact support.

Theorem 2.2 ([10, Theorem 4]). Let $X(\mathbb{R})$ be a separable Banach function space such that the Hardy-Littlewood maximal operator $\mathcal{M}$ is bounded on $X(\mathbb{R})$. Then the set $\mathcal{S}_{0}(\mathbb{R})$ is dense in the space $X(\mathbb{R})$.

\subsection{Banach algebra $\mathcal{M}_{X(\mathbb{R})}$ of Fourier multipliers}

The following result plays an important role in this paper.

Theorem 2.3 ([21, Corollary 4.2] and [8, Theorem 2.4]). Let $X(\mathbb{R})$ be a separable Banach function space such that the Hardy-Littlewood maximal operator $\mathcal{M}$ is bounded on $X(\mathbb{R})$ and on its associate space $X^{\prime}(\mathbb{R})$. If $a \in \mathcal{M}_{X(\mathbb{R})}$, then

$$
\|a\|_{L^{\infty}(\mathbb{R})} \leq\|a\|_{\mathcal{M}_{X(\mathbb{R})}} .
$$

The constant 1 on the right-hand side of (2.1) is best possible.

Inequality (2.1) was established earlier in [18, Theorem 1] with some constant on the right-hand side that depends on the space $X(\mathbb{R})$.

Since (2.1) is available, an easy adaptation of the proof of [12, Proposition 2.5.13] leads to the following (we refer to the proof of [18, Corollary 1] for details). 
Corollary 2.4. Let $X(\mathbb{R})$ be a separable Banach function space such that the Hardy-Littlewood maximal operator $\mathcal{M}$ is bounded on $X(\mathbb{R})$ and on its associate space $X^{\prime}(\mathbb{R})$. Then the set of Fourier multipliers $\mathcal{M}_{X(\mathbb{R})}$ is a Banach algebra under pointwise operations and the norm $\|\cdot\|_{\mathcal{M}_{X(\mathbb{R})}}$.

\subsection{Stechkin-type inequality}

Let $V(\mathbb{R})$ be the Banach algebra of all functions $a: \mathbb{R} \rightarrow \mathbb{C}$ with finite total variation

$$
V(a):=\sup \sum_{i=1}^{n}\left|a\left(t_{i}\right)-a\left(t_{i-1}\right)\right|,
$$

where the supremum is taken over all finite partitions

$$
-\infty<t_{0}<t_{1}<\cdots<t_{n}<+\infty
$$

of the real line $\mathbb{R}$ and the norm in $V(\mathbb{R})$ is given by

$$
\|a\|_{V}=\|a\|_{L^{\infty}(\mathbb{R})}+V(a) .
$$

Theorem 2.5. Let $X(\mathbb{R})$ be a separable Banach function space such that the Hardy-Littlewood maximal operator $\mathcal{M}$ is bounded on $X(\mathbb{R})$ and on its associate space $X^{\prime}(\mathbb{R})$. If $a \in V(\mathbb{R})$, then the convolution operator $W^{0}(a)$ is bounded on the space $X(\mathbb{R})$ and

$$
\left\|W^{0}(a)\right\|_{\mathcal{B}(X(\mathbb{R}))} \leq c_{X}\|a\|_{V}
$$

where $c_{X}$ is a positive constant depending only on $X(\mathbb{R})$.

This result follows from [17, Theorem 4.3].

For Lebesgue spaces $L^{p}(\mathbb{R}), 1<p<\infty$, inequality (2.2) is usually called Stechkin's inequality, and the constant $c_{L^{p}}$ is calculated explicitly:

$$
c_{L^{p}}=\|S\|_{\mathcal{B}\left(L^{p}(\mathbb{R})\right)}= \begin{cases}\tan \left(\frac{\pi}{2 p}\right) & \text { if } \quad 1<p \leq 2, \\ \cot \left(\frac{\pi}{2 p}\right) & \text { if } \quad 2 \leq p<\infty,\end{cases}
$$

where $S$ is the Cauchy singular integral operator given by

$$
(S f)(x):=\frac{1}{\pi i} \lim _{\varepsilon \rightarrow 0} \int_{\mathbb{R} \backslash(x-\varepsilon, x+\varepsilon)} \frac{f(t)}{t-x} d t .
$$

We refer to [7, Theorem 2.11] for the proof of (2.2) in the case of Lebesgue spaces $L^{p}(\mathbb{R})$ with $c_{L^{p}}=\|S\|_{\mathcal{B}\left(L^{p}(\mathbb{R})\right)}$ and to [13, Chap. 13, Theorem 1.3] for the calculation of the norm of $S$ given in the second equality in (2.3). For Lebesgue spaces with Muckenhoupt weights $L^{p}(\mathbb{R}, w)$, the proof of Theorem 2.5 with $c_{L^{p}(w)}=\|S\|_{\mathcal{B}\left(L^{p}(\mathbb{R}, w)\right)}$ is contained in [5, Theorem 17.1]. Further, for variable Lebesgue spaces $L^{p(\cdot)}(\mathbb{R})$, Theorem 2.5 with $c_{L^{p(\cdot)}}=$ $\|S\|_{\mathcal{B}\left(L^{p(\cdot)}(\mathbb{R})\right)}$ was obtained in [20, Theorem 2]. 


\subsection{Slowly oscillating functions}

Let $\dot{\mathbb{R}}=\mathbb{R} \cup\{\infty\}$. For a set $E \subset \dot{\mathbb{R}}$ and a function $f: \dot{\mathbb{R}} \rightarrow \mathbb{C}$ in $L^{\infty}(\mathbb{R})$, let the oscillation of $f$ over $E$ be defined by

$$
\operatorname{osc}(f, E):=\operatorname{essip}_{s, t \in E}|f(s)-f(t)| \text {. }
$$

Following [3, Section 4] and [24, Section 2.1], 25, Section 2.1], we say that a function $f \in L^{\infty}(\mathbb{R})$ is slowly oscillating at a point $\lambda \in \mathbb{R}$ if for every $r \in(0,1)$ or, equivalently, for some $r \in(0,1)$, one has

$$
\begin{array}{ll}
\lim _{x \rightarrow 0+} \operatorname{osc}(f, \lambda+([-x,-r x] \cup[r x, x]))=0 & \text { if } \quad \lambda \in \mathbb{R}, \\
\lim _{x \rightarrow+\infty} \operatorname{osc}(f,[-x,-r x] \cup[r x, x])=0 & \text { if } \quad \lambda=\infty .
\end{array}
$$

For every $\lambda \in \dot{\mathbb{R}}$, let $S O_{\lambda}$ denote the $C^{*}$-subalgebra of $L^{\infty}(\mathbb{R})$ defined by

$$
S O_{\lambda}:=\left\{f \in C_{b}(\dot{\mathbb{R}} \backslash\{\lambda\}): f \text { slowly oscillates at } \lambda\right\},
$$

where $C_{b}(\dot{\mathbb{R}} \backslash\{\lambda\}):=C(\dot{\mathbb{R}} \backslash\{\lambda\}) \cap L^{\infty}(\mathbb{R})$.

Let $S O^{\diamond}$ be the smallest $C^{*}$-subalgebra of $L^{\infty}(\mathbb{R})$ that contains all the $C^{*}$-algebras $S O_{\lambda}$ with $\lambda \in \dot{\mathbb{R}}$. The functions in $S O^{\diamond}$ are called slowly oscillating functions.

\subsection{Banach $S O_{\lambda}^{3}$ of three times continuously differentiable slowly oscillating functions}

For a point $\lambda \in \dot{\mathbb{R}}$, let $C^{3}(\mathbb{R} \backslash\{\lambda\})$ be the set of all three times continuously differentiable functions $a: \mathbb{R} \backslash\{\lambda\} \rightarrow \mathbb{C}$. Following [24, Section 2.4] and 25. Section 2.3], consider the commutative Banach algebras

$$
S O_{\lambda}^{3}:=\left\{a \in S O_{\lambda} \cap C^{3}(\mathbb{R} \backslash\{\lambda\}): \lim _{x \rightarrow \lambda}\left(D_{\lambda}^{k} a\right)(x)=0, k=1,2,3\right\}
$$

equipped with the norm

$$
\|a\|_{S O_{\lambda}^{3}}:=\sum_{j=0}^{3} \frac{1}{j !}\left\|D_{\lambda}^{k} a\right\|_{L^{\infty}(\mathbb{R})},
$$

where $\left(D_{\lambda} a\right)(x)=(x-\lambda) a^{\prime}(x)$ for $\lambda \in \mathbb{R}$ and $\left(D_{\lambda} a\right)(x)=x a^{\prime}(x)$ for $\lambda=\infty$.

Lemma 2.6. For every $\lambda \in \dot{\mathbb{R}}$, the set $S O_{\lambda}^{3}$ is dense in the $C^{*}$-algebra $S O_{\lambda}$.

Proof. In view of [2, Lemma 2.3], the set

$$
S O_{\infty}^{\infty}:=\left\{f \in S O_{\infty} \cap C_{b}^{\infty}(\mathbb{R}): \lim _{x \rightarrow \infty}\left(D_{\infty}^{k} f\right)(x)=0, k \in \mathbb{N}\right\}
$$

is dense in the Banach algebra $S O_{\infty}$. Here $C_{b}^{\infty}(\mathbb{R})$ denotes the set of all infinitely differentiable functions $f: \mathbb{R} \rightarrow \mathbb{C}$, which are bounded with all their derivatives. Note that $S O_{\infty}^{\infty}$ can be equivalently defined by replacing $C_{b}^{\infty}$ in (2.4) by $C^{\infty}$, because $f \in S O_{\infty}$ is bounded and its derivatives $f^{(k)}$ are bounded for all $k \in \mathbb{N}$ in view of $\lim _{x \rightarrow \infty}\left(D_{\infty}^{k} f\right)(x)=0$. Since $S O_{\infty}^{\infty} \subset S O_{\infty}^{3}$, this completes the proof in the case $\lambda=\infty$. 
If $\lambda \in \mathbb{R}$, then by [25. Corollary 2.2], the mapping $T a=a \circ \beta_{\lambda}$, where $\beta_{\lambda}: \dot{\mathbb{R}} \rightarrow \dot{\mathbb{R}}$ is defined by

$$
\beta_{\lambda}(x)=\frac{\lambda x-1}{x+\lambda}
$$

is an isometric isomorphism of the algebra $\mathrm{SO}_{\lambda}$ onto the algebra $\mathrm{SO}_{\infty}$. Hence each function $a \in S O_{\lambda}$ can be approximated in the norm of $S O_{\lambda}$ by functions $c_{n}=b_{n} \circ \beta_{\lambda}^{-1}$, where $b_{n} \in S O_{\infty}^{\infty}$ for $n \in \mathbb{N}$ and

$$
\beta_{\lambda}^{-1}(y)=\frac{\lambda y+1}{\lambda-y}=x, \quad x, y \in \dot{\mathbb{R}} .
$$

It remains to show that $c_{n} \in S O_{\lambda}^{3}$. Taking into account (2.5)-(2.6), we obtain for $y=\beta_{\lambda}(x) \in \mathbb{R} \backslash\{\lambda\}$ and $x=\beta_{\lambda}^{-1}(y) \in \mathbb{R}$ :

$$
\begin{aligned}
\left(D_{\lambda} c_{n}\right)(y) & =b_{n}^{\prime}\left(\beta_{\lambda}^{-1}(y)\right) \frac{\lambda^{2}+1}{y-\lambda}=-b_{n}^{\prime}(x)(x+\lambda), \\
\left(D_{\lambda}^{2} c_{n}\right)(y) & =b_{n}^{\prime \prime}\left(\beta_{\lambda}^{-1}(y)\right) \frac{\left(\lambda^{2}+1\right)^{2}}{y-\lambda}-b_{n}^{\prime}\left(\beta_{\lambda}^{-1}(y)\right) \frac{\lambda^{2}+1}{y-\lambda} \\
& =-b_{n}^{\prime \prime}(x)(x+\lambda)\left(\lambda^{2}+1\right)+b_{n}^{\prime}(x)(x+\lambda), \\
\left(D_{\lambda}^{3} c_{n}\right)(y)= & b_{n}^{\prime \prime \prime}\left(\beta_{\lambda}^{-1}(y)\right) \frac{\left(\lambda^{2}+1\right)^{3}}{y-\lambda}-2 b_{n}^{\prime \prime}\left(\beta_{\lambda}^{-1}(y)\right) \frac{\left(\lambda^{2}+1\right)^{2}}{y-\lambda} \\
& +b_{n}^{\prime}\left(\beta_{\lambda}^{-1}(y)\right) \frac{\lambda^{2}+1}{y-\lambda} \\
= & -b_{n}^{\prime \prime \prime}(x)(x+\lambda)\left(\lambda^{2}+1\right)^{2}+2 b_{n}^{\prime \prime}(x)(x+\lambda)\left(\lambda^{2}+1\right) \\
& -b_{n}^{\prime}(x)(x+\lambda) .
\end{aligned}
$$

Since

we see that

$$
\lim _{x \rightarrow \infty}\left(D_{\infty}^{k} b_{n}\right)(x)=0 \quad \text { for } \quad k \in\{1,2,3\}
$$

$$
\lim _{x \rightarrow \infty} x^{k} b_{n}^{(k)}(x)=0 \quad \text { for } \quad k \in\{1,2,3\} .
$$

It follows from (2.7)-2.10) that

$$
\lim _{y \rightarrow \lambda}\left(D_{\lambda}^{k} c_{n}\right)(y)=0 \quad \text { for } \quad k \in\{1,2,3\} .
$$

Hence $c_{n} \in S O_{\lambda}^{3}$ for all $n \in \mathbb{N}$, which completes the proof.

\subsection{Slowly oscillating Fourier multipliers}

The following result leads us to the definition of slowly oscillating Fourier multipliers.

Theorem 2.7 ([19, Theorem 2.5]). Let $X(\mathbb{R})$ be a separable Banach function space such that the Hardy-Littlewood maximal operator $\mathcal{M}$ is bounded on $X(\mathbb{R})$ and on its associate space $X^{\prime}(\mathbb{R})$. If $\lambda \in \dot{\mathbb{R}}$ and $a \in S O_{\lambda}^{3}$, then the convolution operator $W^{0}(a)$ is bounded on the space $X(\mathbb{R})$ and

$$
\left\|W^{0}(a)\right\|_{\mathcal{B}(X(\mathbb{R}))} \leq c_{X}\|a\|_{S O_{\lambda}^{3}}
$$

where $c_{X}$ is a positive constant depending only on $X(\mathbb{R})$. 
Let $S O_{\lambda, X(\mathbb{R})}$ denote the closure of $S O_{\lambda}^{3}$ in the norm of $\mathcal{M}_{X(\mathbb{R})}$. Further, let $S O_{X(\mathbb{R})}^{\diamond}$ be the smallest Banach subalgebra of $\mathcal{M}_{X(\mathbb{R})}$ that contains all the Banach algebras $S O_{\lambda, X(\mathbb{R})}$ for $\lambda \in \dot{\mathbb{R}}$. The functions in $S O_{X(\mathbb{R})}^{\diamond}$ will be called slowly oscillating Fourier multipliers.

Lemma 2.8. Let $X(\mathbb{R})$ be a separable Banach function space such that the Hardy-Littlewood maximal operator $\mathcal{M}$ is bounded on $X(\mathbb{R})$ and on its associate space $X^{\prime}(\mathbb{R})$. Then

$$
S O_{X(\mathbb{R})}^{\diamond} \subset S O_{L^{2}(\mathbb{R})}^{\diamond}=S O^{\diamond} .
$$

Proof. The continuous embedding $S O_{X(\mathbb{R})}^{\diamond} \subset S O_{L^{2}(\mathbb{R})}^{\diamond}$ (with the embedding constant one) follows immediately from Theorem 2.3 and the definitions of the Banach algebras $S O_{X(\mathbb{R})}^{\diamond}$ and $S O_{L^{2}(\mathbb{R})}^{\diamond}$. It is clear that $S O_{L^{2}(\mathbb{R})}^{\diamond} \subset S O^{\diamond}$. The embedding $S O^{\diamond} \subset S O_{L^{2}(\mathbb{R})}^{\diamond}$ follows from Lemma 2.6.

\section{Maximal ideal spaces of the algebras $S O^{\diamond}$ and $S O_{X(\mathbb{R})}^{\diamond}$}

\subsection{Extensions of multiplicative linear functionals on $C^{*}$-algebras}

For a $C^{*}$-algebra (or, more generally, a Banach algebra) $\mathfrak{A}$ with unit $e$ and an element $a \in \mathfrak{A}$, let $\operatorname{sp}_{\mathfrak{A}}(a)$ denote the spectrum of $a$ in $\mathfrak{A}$. Recall that an element $a$ of a $C^{*}$-algebra $\mathfrak{A}$ is said to be positive if it is self-adjoint and $\operatorname{sp}_{\mathfrak{A}}(a) \subset[0, \infty)$. A linear functional $\phi$ on $\mathfrak{A}$ is said to be a state if $\phi(a) \geq 0$ for all positive elements $a \in \mathfrak{A}$ and $\phi(e)=1$. The set of all states of $\mathfrak{A}$ is denoted by $\mathfrak{S}(\mathfrak{A})$. The extreme points of $\mathfrak{S}(\mathfrak{A})$ are called pure states of $\mathfrak{A}$ (see, e.g., [15, Section 4.3]).

Following [1, p. 304], for a state $\phi$, let

$$
\mathcal{G}_{\phi}(\mathfrak{A}):=\left\{a \in \mathfrak{A}:|\phi(a)|=\|a\|_{\mathfrak{A}}=1\right\}
$$

and let $\mathcal{G}_{\phi}^{+}(\mathfrak{A})$ denote the set of all positive elements of $\mathcal{G}_{\phi}(\mathfrak{A})$. Let $\mathfrak{A}$ and $\mathfrak{B}$ be $C^{*}$-algebras such that $e \in \mathfrak{B} \subset \mathfrak{A}$. Let $\phi$ be a state of $\mathfrak{B}$. Following [1, p. 310], we say that $\mathfrak{A}$ is $\mathfrak{B}$-compressible modulo $\phi$ if for each $x \in \mathfrak{A}$ and each $\varepsilon>0$ there is $b \in \mathcal{G}_{\phi}^{+}(\mathfrak{B})$ and $y \in \mathfrak{B}$ such that $\|b x b-y\|_{\mathfrak{A}}<\varepsilon$.

Since a nonzero linear functional on a commutative $C^{*}$-algebra is a pure state if and only if it is multiplicative (see, e.g., [15, Proposition 4.4.1]), we immediately get the following lemma from [1, Theorem 3.2].

Lemma 3.1. Let $\mathfrak{B}$ be a $C^{*}$-subalgebra of a commutative $C^{*}$-algebra $\mathfrak{A}$. $A$ nonzero multiplicative linear functional $\phi$ on $\mathfrak{B}$ admits a unique extension to a multiplicative linear functional $\phi^{\prime}$ on $\mathfrak{A}$ if and only if $\mathfrak{A}$ is $\mathfrak{B}$-compressible modulo $\phi$.

\subsection{Family of positive elements}

For $t \in \dot{\mathbb{R}}$ and $\omega>0$, let $\psi_{t, \omega}$ be a real-valued function in $C(\dot{\mathbb{R}})$ such that $0 \leq \psi_{t, \omega}(x) \leq 1$ for all $x \in \mathbb{R}$. Assume that for $t \in \mathbb{R}$,

$\psi_{t, \omega}(s)=1 \quad$ if $\quad s \in(t-\omega, t+\omega), \quad \psi_{t, \omega}(s)=0 \quad$ if $\quad s \in \mathbb{R} \backslash(t-2 \omega, t+2 \omega)$, 
and for $t=\infty$,

$$
\psi_{\infty, \omega}(s)=1 \quad \text { if } \quad s \in \mathbb{R} \backslash(-2 \omega, 2 \omega), \quad \psi_{\infty, \omega}(s)=0 \quad \text { if } \quad s \in(-\omega, \omega) .
$$

Let $M(\mathfrak{A})$ denote the maximal ideal space of a commutative Banach algebra $\mathfrak{A}$.

Lemma 3.2. For $t \in \dot{\mathbb{R}}$ and $\omega>0$, the function $\psi_{t, \omega}$ is a positive element of the $C^{*}$-algebras $C(\dot{\mathbb{R}}), S O_{t}$, and $S O^{\diamond}$.

Proof. Since $M(C(\dot{\mathbb{R}}))=\dot{\mathbb{R}}$, it follows from the Gelfand theorem (see, e.g., 28. Theorem 2.1.3]) that $\operatorname{sp}_{C(\dot{\mathbb{R}})}\left(\psi_{t, \omega}\right)=[0,1]$ for all $t \in \dot{\mathbb{R}}$ and all $\omega>0$. Since $C(\dot{\mathbb{R}}) \subset S O_{t} \subset S O^{\diamond}$, we conclude that the functions $\psi_{t, \omega}$ for $t \in \dot{\mathbb{R}}$ and $\omega>0$ are positive elements of the $C^{*}$-algebras $C(\dot{\mathbb{R}}), S O_{t}$, and $S O^{\diamond}$ because their spectra in each of these algebras coincide with $[0,1]$ in view of [15. Proposition 4.1.5].

\subsection{Maximal ideal space of the $C^{*}$-algebra $S O^{\diamond}$}

If $\mathfrak{B}$ is a Banach subalgebra of $\mathfrak{A}$ and $\lambda \in M(\mathfrak{B})$, then the set

$$
M_{\lambda}(\mathfrak{A}):=\left\{\xi \in M(\mathfrak{A}):\left.\xi\right|_{\mathfrak{B}}=\lambda\right\}
$$

is called the fiber of $M(\mathfrak{A})$ over $\lambda \in M(\mathfrak{B})$. Hence for every Banach algebra $\Phi \subset L^{\infty}(\mathbb{R})$ with $M(C(\dot{\mathbb{R}}) \cap \Phi)=\dot{\mathbb{R}}$ and every $t \in \dot{\mathbb{R}}$, the fiber $M_{t}(\Phi)$ is the set of all multiplicative linear functionals (characters) on $\Phi$ that annihilate the set $\{f \in C(\dot{\mathbb{R}}) \cap \Phi: f(t)=0\}$. As usual, for all $a \in \Phi$ and all $\xi \in M(\Phi)$, we put $a(\xi):=\xi(a)$. We will frequently identify the points $t \in \dot{\mathbb{R}}$ with the evaluation functionals $\delta_{t}$ defined by

$$
\delta_{t}(f)=f(t) \quad \text { for } \quad f \in C(\dot{\mathbb{R}}), \quad t \in \dot{\mathbb{R}} .
$$

Lemma 3.3. For every point $t \in \dot{\mathbb{R}}$, the fibers $M_{t}\left(S O_{t}\right)$ and $M_{t}\left(S O^{\diamond}\right)$ can be identified as sets:

$$
M_{t}\left(S O_{t}\right)=M_{t}\left(S O^{\diamond}\right) .
$$

Proof. Since $C(\dot{\mathbb{R}}) \subset S O_{t} \subset S O^{\diamond}$, by the restriction of a multiplicative linear functional defined on a bigger algebra to a smaller algebra, we have

$$
M\left(S O^{\diamond}\right) \subset M\left(S O_{t}\right) \subset M(C(\dot{\mathbb{R}})), \quad t \in \dot{\mathbb{R}} .
$$

Since

$$
M(\Phi)=\bigcup_{t \in \mathbb{R}} M_{t}(\Phi) \quad \text { for } \quad \Phi \in\left\{S O^{\diamond}, S O_{\lambda}: \lambda \in \dot{\mathbb{R}}\right\}
$$

where

$$
M_{t}(\Phi)=\left\{\zeta \in M(\Phi):\left.\zeta\right|_{C(\dot{\mathbb{R}})}=\delta_{t}\right\}, \quad t \in \dot{\mathbb{R}},
$$

it follows from (3.2) and (3.3) that

$$
M_{t}\left(S O^{\diamond}\right) \subset M_{t}\left(S O_{t}\right), \quad t \in \mathbb{R} .
$$

Now fix $t \in \dot{\mathbb{R}}$ and a multiplicative linear functional $\eta \in M_{t}\left(S O_{t}\right)$. Let us show that the $C^{*}$-algebra $S O^{\diamond}$ is $S O_{t}$-compressible modulo $\eta$. Take $\varepsilon>0$. 
By the definition of $S O^{\diamond}$, for a function $x \in S O^{\diamond}$, there are a finite set $F \in \dot{\mathbb{R}}$ and a finite set $\left\{x_{\lambda} \in S O_{\lambda}: \lambda \in F\right\}$ such that

$$
\left\|x-\sum_{\lambda \in F} x_{\lambda}\right\|_{L^{\infty}(\mathbb{R})}<\varepsilon .
$$

If $t \neq \infty$, take $\omega$ such that

$$
0<\omega<\frac{1}{2} \min _{\lambda \in F \backslash\{t\}}|\lambda-t|
$$

and $b:=\psi_{t, \omega}$. Then

$$
y:=b\left(\sum_{\lambda \in F} x_{\lambda}\right) b
$$

is equal to zero outside the interval $(t-2 \omega, t+2 \omega)$. Therefore, $y \in S O_{t}$.

If $t=\infty$, take $\omega$ such that

$$
\omega>\max _{\lambda \in F \backslash\{\infty\}}|\lambda|
$$

and $b:=\psi_{\infty, \omega}$. Then the function $y$ defined by (3.5) is equal to zero on $(-\omega, \omega)$ and $y \in S O_{\infty}$.

For $t \in \dot{\mathbb{R}}$, we have

$$
\|b x b-y\|_{L^{\infty}(\mathbb{R})}=\left\|b\left(x-\sum_{\lambda \in F} x_{\lambda}\right) b\right\|_{L^{\infty}(\mathbb{R})} \leq\left\|x-\sum_{\lambda \in F} x_{\lambda}\right\|_{L^{\infty}(\mathbb{R})}<\varepsilon .
$$

Since $b$ is a positive element of $S O_{t}$ in view of Lemma 3.2. we have $b \in$ $\mathcal{G}_{\eta}^{+}\left(S O_{t}\right)$, which completes the proof of the fact that $S O^{\diamond}$ is $S O_{t}$-compressible modulo the multiplicative linear functional $\eta \in M_{t}\left(S O_{t}\right)$.

In view of Lemma 3.1. there exists a unique extension $\eta^{\prime}$ of the multiplicative linear functional $\eta$ to the whole algebra $S O^{\diamond}$. By the definition of the fiber $M_{t}\left(S O^{\diamond}\right)$, we have $\eta^{\prime} \in M_{t}\left(S O^{\diamond}\right)$. Thus, we can identify $M_{t}\left(S O_{t}\right)$ with a subset of $M_{t}\left(S O^{\diamond}\right)$ :

$$
M_{t}\left(S O_{t}\right) \subset M_{t}\left(S O^{\diamond}\right) .
$$

Combining (3.4) and (3.6), we arrive at (3.1).

Corollary 3.4. The maximal ideal space of the commutative $C^{*}$-algebra $S O^{\diamond}$ can be identified with the set

$$
\bigcup_{t \in \mathbb{R}} M_{t}\left(S O_{t}\right)
$$

\subsection{Extensions of multiplicative linear functionals on Banach algebras}

The following theorem in a slightly different form is contained in [29, Theorem 2.1.1] and [30, Theorem 3.10]. For the convenience of readers, we give its proof here. 
Theorem 3.5. Let $\mathfrak{A}, \mathfrak{B}, \mathfrak{C}$ be commutative unital Banach algebras with common unit and homomorphic imbeddings $\mathfrak{A} \subset \mathfrak{B} \subset \mathfrak{C}$, where $\mathfrak{A}$ is dense in $\mathfrak{B}$. If for each functional $\varphi \in M(\mathfrak{A})$ there exists a unique extension $\varphi^{\prime} \in M(\mathfrak{C})$, then for every functional $\psi \in M(\mathfrak{B})$ there exists a unique extension $\psi^{\prime} \in M(\mathfrak{C})$.

Proof. Let $\psi \in M(\mathfrak{B})$. Then $\psi_{1}:=\left.\psi\right|_{\mathfrak{A}} \in M(\mathfrak{A})$. By the hypotheses, there exists a unique extension $\psi_{3}:=\left(\psi_{1}\right)^{\prime} \in M(\mathfrak{C})$. Then $\psi_{1}(a)=\psi(a)=\psi_{3}(a)$ for all $a \in \mathfrak{A}$. Let $\psi_{2}:=\left.\psi_{3}\right|_{\mathfrak{B}} \in M(\mathfrak{B})$. Since $\mathfrak{A} \subset \mathfrak{B}$, it follows that

$$
\psi(a)=\psi_{2}(a) \text { for all } a \in \mathfrak{A} .
$$

On the other hand, functionals $\psi, \psi_{2} \in M(\mathfrak{B})$ are continuous on $\mathfrak{B}$ (see, e.g., [16. Lemma 2.1.5]). Since $\mathfrak{A}$ is dense in $\mathfrak{B}$, for every $b \in \mathfrak{B}$ there exists a sequence $\left\{a_{n}\right\}_{n \in \mathbb{N}} \subset \mathfrak{A}$ such that $\left\|a_{n}-b\right\|_{\mathfrak{B}} \rightarrow 0$ as $n \rightarrow \infty$. It follows from this observation and (3.7) that for every $b \in \mathfrak{B}$,

$$
\psi(b)=\lim _{n \rightarrow \infty} \psi\left(a_{n}\right)=\lim _{n \rightarrow \infty} \psi_{2}\left(a_{n}\right)=\psi_{2}(b)=\psi_{3}(b) .
$$

Thus $\psi_{3} \in M(\mathfrak{C})$ is an extension of $\psi$. This extension is unique by construction.

\subsection{Maximal ideal space of the Banach algebras $S O_{t, X}(\mathbb{R})$}

We start with the following refinement of [25, Lemma 3.4].

Lemma 3.6. Let $t \in \dot{\mathbb{R}}$. Then for each functional $\varphi \in M\left(S O_{t}^{3}\right)$ there exists a unique extension $\varphi^{\prime} \in M\left(S O_{t}\right)$.

The density of $S O_{t}^{3}$ in the Banach algebra $S O_{t}$ essentially used in the proof of [25, Lemma 3.4] is justified in Lemma 2.6. Note that the uniqueness of an extension was not explicitly mentioned in [25, Lemma 3.4]. However, since $M\left(S O_{t}^{3}\right)$ and $M\left(S O_{t}\right)$ are Hausdorff spaces (see, e.g., [16, Theorem 2.2.3]), the uniqueness of an extension constructed in the proof of [25. Lemma 3.4] is a consequence of a standard fact from general topology (see, e.g,, [27. Theorem IV.2(b)]).

The following lemma is analogous to [25, Lemma 3.5].

Lemma 3.7. Let $X(\mathbb{R})$ be a separable Banach function space such that the Hardy-Littlewood maximal operator $\mathcal{M}$ is bounded on the space $X(\mathbb{R})$ and on its associate space $X^{\prime}(\mathbb{R})$. If $t \in \dot{\mathbb{R}}$, then the maximal ideal spaces of the $C^{*}$-algebra $S O_{t}$ and the Banach algebra $S O_{t, X(\mathbb{R})}$ can be identified as sets:

$$
M\left(S O_{t}\right)=M\left(S O_{t, X(\mathbb{R})}\right) .
$$

Proof. It follows from Theorem 2.3 that $S O_{t}^{3} \subset S O_{t, X(\mathbb{R})} \subset S O_{t}$, where the imbeddings are homomorphic. By the definition of the algebra $S O_{t, X(\mathbb{R})}$, the algebra $S O_{t}^{3}$ is dense in $S O_{t, X(\mathbb{R})}$ with respect to the norm of $\mathcal{M}_{X(\mathbb{R})}$. Taking into account these observations and Lemma 3.6 we see that the commutative Banach algebras

$$
\mathfrak{A}=S O_{t}^{3}, \quad \mathfrak{B}=S O_{t, X(\mathbb{R})}, \quad \mathfrak{C}=S O_{t}
$$


satisfy all the conditions of Theorem 3.5. By this theorem, every multiplicative linear functional on $S O_{t, X(\mathbb{R})}$ admits a unique extension to a multiplicative linear functional on $S O_{t}$. Hence we can identify $M\left(S O_{t, X(\mathbb{R})}\right)$ with a subset of $M\left(S O_{t}\right)$ :

$$
M\left(S O_{t, X(\mathbb{R})}\right) \subset M\left(S O_{t}\right) .
$$

On the other hand, since $S O_{t, X(\mathbb{R})} \subset S O_{t}$, by the restriction of a multiplicative linear functional defined on a bigger algebra to a smaller algebra, we have

$$
M\left(S O_{t}\right) \subset M\left(S O_{t, X(\mathbb{R})}\right) .
$$

Combining inclusions (3.9) and (3.10), we immediately arrive at (3.8).

The next lemma is analogous to Lemma 3.3

Lemma 3.8. Let $X(\mathbb{R})$ be a separable Banach function space such that the Hardy-Littlewood maximal operator $\mathcal{M}$ is bounded on the space $X(\mathbb{R})$ and on its associate space $X^{\prime}(\mathbb{R})$. Then, for every point $t \in \dot{\mathbb{R}}$, the fibers $M_{t}\left(S O_{t, X(\mathbb{R})}\right)$ and $M_{t}\left(S O_{X(\mathbb{R})}^{\diamond}\right)$ can be identified as sets:

$$
M_{t}\left(S O_{t, X(\mathbb{R})}\right)=M_{t}\left(S O_{X(\mathbb{R})}^{\diamond}\right)
$$

Proof. Since $S O_{t, X(\mathbb{R})} \subset S O_{X(\mathbb{R})}^{\diamond}$ for every $t \in \dot{\mathbb{R}}$, we conclude by the restriction of a multiplicative linear functional defined on the bigger algebra to the smaller algebra that $M\left(S O_{X(\mathbb{R})}^{\diamond}\right) \subset M\left(S O_{t, X(\mathbb{R})}\right)$. Hence

$$
M_{t}\left(S O_{X(\mathbb{R})}^{\diamond}\right) \subset M_{t}\left(S O_{t, X(\mathbb{R})}\right) .
$$

On the other hand, in view of Lemma 3.7. any multiplicative linear functional $\xi \in M_{t}\left(S O_{t, X(\mathbb{R})}\right)$ admits a unique extension $\xi^{\prime} \in M\left(S O_{t}\right)$. Moreover, $\xi^{\prime}$ belongs to $M_{t}\left(S O_{t}\right)$ as well. By Lemma 3.3. the functional $\xi^{\prime} \in M_{t}\left(S O_{t}\right)$ admits a unique extension $\xi^{\prime \prime} \in M_{t}\left(S O^{\diamond}\right)$. It is clear that the restriction of $\xi^{\prime \prime}$ to $S O_{X(\mathbb{R})}^{\diamond}$ belongs to $M_{t}\left(S O_{X(\mathbb{R})}^{\diamond}\right)$. Thus $M_{t}\left(S O_{t, X(\mathbb{R})}\right)$ can be identified with a subset of $M_{t}\left(S O_{X(\mathbb{R})}^{\diamond}\right)$ :

$$
M_{t}\left(S O_{t, X(\mathbb{R})}\right) \subset M_{t}\left(S O_{X(\mathbb{R})}^{\diamond}\right) .
$$

Combining (3.12) and (3.13), we arrive at (3.11).

\subsection{Maximal ideal space of the Banach algebra $S O_{X(\mathbb{R})}^{\diamond}$}

Now we are in a position to prove that the maximal ideal spaces of the commutative Banach algebra $S O_{X(\mathbb{R})}^{\diamond}$ and the $C^{*}$-algebra $S O^{\diamond}$ can be identified as sets.

Theorem 3.9. Let $X(\mathbb{R})$ be a separable Banach function space such that the Hardy-Littlewood maximal operator $\mathcal{M}$ is bounded on the space $X(\mathbb{R})$ and on its associate space $X^{\prime}(\mathbb{R})$. Then the maximal ideal space of the Banach algebra $S O_{X(\mathbb{R})}^{\diamond}$ can be identified with the maximal ideal space of the $C^{*}$-algebra $S O^{\diamond}$ :

$$
M\left(S O_{X(\mathbb{R})}^{\diamond}\right)=M\left(S O^{\diamond}\right) .
$$


Proof. It follows from Lemmas 3.8, 3.7 and 3.3 that for every $t \in \dot{\mathbb{R}}$,

$$
M_{t}\left(S O_{X(\mathbb{R})}^{\diamond}\right)=M_{t}\left(S O_{t, X(\mathbb{R})}\right)=M_{t}\left(S O_{t}\right)=M_{t}\left(S O^{\diamond}\right) .
$$

Hence

$$
M\left(S O_{X(\mathbb{R})}^{\diamond}\right)=\bigcup_{t \in \mathbb{R}} M_{t}\left(S O_{X(\mathbb{R})}^{\diamond}\right)=\bigcup_{t \in \mathbb{R}} M_{t}\left(S O^{\diamond}\right)=M\left(S O^{\diamond}\right),
$$

which completes the proof.

\section{Maximal ideal spaces of the Calkin images of the Banach algebras $\mathcal{M O}(\Phi)$ and $\mathcal{C O}(\Psi)$}

\subsection{Maximal ideal space of the algebra $\mathcal{M O}^{\pi}(\Phi)$}

We start with the following known result [14, Theorem 2.4] (see also 9, Theorem 3.1]).

Theorem 4.1. Let $X(\mathbb{R})$ be a separable Banach function space and $a \in L^{\infty}(\mathbb{R})$. Then the multiplication operator aI is compact on the space $X(\mathbb{R})$ if and only if $a=0$ almost everywhere on $\mathbb{R}$.

The next theorem says that one can identify the maximal ideal spaces of the algebras $\mathcal{M O}^{\pi}(\Phi)$ and $\Phi$ for an arbitrary unital $C^{*}$-subalgebra of $L^{\infty}(\mathbb{R})$.

Theorem 4.2. Let $X(\mathbb{R})$ be a separable Banach function space. If $\Phi$ is a unital $C^{*}$-subalagebra of $L^{\infty}(\mathbb{R})$, then the maximal ideal spaces of the commutative Banach algebra $\mathcal{M O}^{\pi}(\Phi)$ and the commutative $C^{*}$-algebra $\Phi$ are homeomorphic:

$$
M\left(\mathcal{M O}^{\pi}(\Phi)\right)=M(\Phi) .
$$

Proof. Consider the mapping $F: \Phi \rightarrow \mathcal{M O}^{\pi}(\Phi)$ defined by $F(a)=[a I]^{\pi}$ for every $a \in \Phi$. It is clear that this mapping is surjective. If $[a I]^{\pi}=[b I]^{\pi}$ for some $a, b \in \Phi$, then $(a-b) I \in \mathcal{K}(X(\mathbb{R}))$. It follows from Theorem 4.1] that $a=b$ a.e. on $\mathbb{R}$. This implies that the mapping $F$ is injective. Thus, $F: \Phi \rightarrow \mathcal{M O}^{\pi}(\Phi)$ is an algebraic isomorphism of commutative Banach algebras. It follows from [16. Lemma 2.2.12] that the maximal ideal spaces $M\left(\mathcal{M O}^{\pi}(\Phi)\right)$ and $M(\Phi)$ are homeomorphic.

\subsection{Maximal ideal space of the algebra $\mathcal{C O}^{\pi}(\Psi)$}

The following analogue of Theorem 4.1 for Fourier convolution operators was obtained recently by the authors [8, Theorem 1.1].

Theorem 4.3. Let $X(\mathbb{R})$ be a separable Banach function space such that the Hardy-Littlewood maximal operator $\mathcal{M}$ is bounded on $X(\mathbb{R})$ and on its associate space $X^{\prime}(\mathbb{R})$. Suppose that $b \in \mathcal{M}_{X(\mathbb{R})}$. Then the Fourier convolution operator $W^{0}(a)$ is compact on the space $X(\mathbb{R})$ if and only if $b=0$ almost everywhere on $\mathbb{R}$.

The next theorem is an analogue of Theorem 4.2 for Fourier multipliers. 
Theorem 4.4. Let $X(\mathbb{R})$ be a separable Banach function space such that the Hardy-Littlewood maximal operator $\mathcal{M}$ is bounded on $X(\mathbb{R})$ and on its associate space $X^{\prime}(\mathbb{R})$. If $\Psi$ is a unital Banach subalagebra of $\mathcal{M}_{X(\mathbb{R})}$, then the maximal ideal spaces of the commutative Banach algebras $\mathcal{C O}^{\pi}(\Psi)$ and $\Psi$ are homeomorphic:

$$
M\left(\mathcal{C O}^{\pi}(\Psi)\right)=M(\Psi)
$$

Proof. The proof is analogous to the proof of Theorem 4.2 Consider the mapping $F: \Psi \rightarrow \mathcal{C O}^{\pi}(\Psi)$ defined by $F(a)=\left[W^{0}(a)\right]^{\pi}$ for every $a \in \Psi$. It is obvious that this mapping is surjective. If $\left[W^{0}(a)\right]^{\pi}=\left[W^{0}(b)\right]^{\pi}$ for some $a, b \in \Psi$, then $W^{0}(a-b)=W^{0}(a)-W^{0}(b) \in \mathcal{K}(X(\mathbb{R}))$. By Theorem 4.3. we conclude that $a=b$ a.e. on $\mathbb{R}$. Therefore, the mapping $F$ is injective. Thus, $F: \Psi \rightarrow \mathcal{C O}^{\pi}(\Psi)$ is an algebraic isomorphism of commutative Banach algebras. In this case it follows from [16, Lemma 2.2.12] that the maximal ideal spaces $M\left(\mathcal{C O}^{\pi}(\Psi)\right)$ and $M(\Psi)$ are homeomorphic.

\section{Applications of the method of limit operators}

\subsection{Known result about limit operators on Banach function spaces}

Let $X(\mathbb{R})$ be a Banach function space. For a sequence of operators $\left\{A_{n}\right\}_{n \in \mathbb{N}} \subset$ $\mathcal{B}(X(\mathbb{R}))$, let

$$
\operatorname{s-lim}_{n \rightarrow \infty} A_{n}
$$

denote the strong limit of this sequence, if it exists. For $\lambda, x \in \mathbb{R}$, consider the function

$$
e_{\lambda}(x):=e^{i \lambda x}
$$

Let $T \in \mathcal{B}(X(\mathbb{R}))$ and let $h=\left\{h_{n}\right\}_{n \in \mathbb{N}}$ be a sequence of numbers $h_{n}>0$ such that $h_{n} \rightarrow+\infty$ as $n \rightarrow \infty$. The strong limit

$$
T_{h}:=\underset{n \rightarrow \infty}{\mathrm{S}-\lim _{n}} e_{h_{n}} T e_{h_{n}}^{-1} I
$$

is called the limit operator of $T$ related to the sequence $h=\left\{h_{n}\right\}_{n \in \mathbb{N}}$, if it exists.

In our previous paper 9] we calculated the limit operators for all compact operators.

Lemma 5.1 (9. Lemma 3.2]). Let $X(\mathbb{R})$ be a separable Banach function space and $K$ be a compact operator on $X(\mathbb{R})$. Then for every sequence $\left\{h_{n}\right\}_{n \in \mathbb{N}}$ of positive numbers satisfying $h_{n} \rightarrow+\infty$ as $n \rightarrow \infty$, one has

$$
\text { S- } \lim _{n \rightarrow \infty} e_{h_{n}} K e_{h_{n}}^{-1} I=0
$$

on the space $X(\mathbb{R})$. 


\subsection{Limit operators for Fourier convolution operators with symbols in the algebra $S O_{X(\mathbb{R})}^{\diamond}$}

Now we will calculate the limit operators for the Fourier convolution operator with a slowly oscillating symbol.

Theorem 5.2. Let $X(\mathbb{R})$ be a separable Banach function space such that the Hardy-Littlewood maximal operator $\mathcal{M}$ is bounded on the space $X(\mathbb{R})$ and on its associate space $X^{\prime}(\mathbb{R})$. If $b \in S O_{X(\mathbb{R})}^{\diamond}$, then for every $\xi \in M_{\infty}\left(S O^{\diamond}\right)$ there exists a sequence $\left\{h_{n}\right\}_{n \in \mathbb{N}}$ of positive numbers such that $h_{n} \rightarrow+\infty$ as $n \rightarrow \infty$ and

$$
\underset{n \rightarrow \infty}{\mathrm{s}-\lim _{n}} e_{h_{n}} W^{0}(b) e_{h_{n}}^{-1} I=b(\xi) I
$$

on the space $X(\mathbb{R})$.

Proof. This statement is proved by analogy with [25, Lemma 5.1]. In view of Lemma 2.8, $S O_{X(\mathbb{R})}^{\diamond} \subset S O^{\diamond}$. Therefore every $\xi \in M_{\infty}\left(S O^{\diamond}\right)$ is a multiplicative linear functional on $S O_{X(\mathbb{R})}^{\diamond}$, that is, $b(\xi)$ is well defined. By the definition of $S O_{X(\mathbb{R})}^{\diamond}$, if $b \in S O_{X(\mathbb{R})}^{\diamond}$, then there is a sequence

$$
b_{m}=\sum_{\lambda \in F_{m}} b_{m, \lambda}, \quad m \in \mathbb{N},
$$

where $F_{m} \subset \dot{\mathbb{R}}$ are finite sets and $b_{m, \lambda} \in S O_{\lambda}^{3}$ for $\lambda \in F_{m}$ and all $m \in \mathbb{N}$, such that

$$
\lim _{m \rightarrow \infty}\left\|b_{m}-b\right\|_{\mathcal{M}_{X(\mathbb{R})}}=0 .
$$

By Lemma 3.3. $M_{\infty}\left(S O^{\diamond}\right)=M_{\infty}\left(S O_{\infty}\right)$. Fix $\xi \in M_{\infty}\left(S O^{\diamond}\right)=M_{\infty}\left(S O_{\infty}\right)$. Assume first that the set

$$
B_{\infty}:=\left\{b_{m, \infty} \in S O_{\infty}^{3}: m \in \mathbb{N}\right\}
$$

is not empty. Since the set $B_{\infty}$ is at most countable, it follows from 2 , Corollary 3.3] or [25, Proposition 3.1] that there exists a sequence $\left\{h_{n}\right\}_{n \in \mathbb{N}}$ such that $h_{n} \rightarrow+\infty$ as $n \rightarrow \infty$ and

$$
\xi\left(b_{m, \infty}\right)=\lim _{n \rightarrow \infty} b_{m, \infty}\left(h_{n}\right) \quad \text { for all } \quad b_{m, \infty} \in B_{\infty}
$$

As the functions $b_{m, \lambda}$ are continuous at $\infty$ if $\lambda \neq \infty$, we see that

$$
\xi\left(b_{m, \lambda}\right)=b_{m, \lambda}(\infty)=\lim _{n \rightarrow \infty} b_{m, \lambda}\left(h_{n}\right) \quad \text { for all } \quad \lambda \in \bigcup_{m \in \mathbb{N}} F_{m} \backslash\{\infty\} .
$$

Combining (5.3) and (5.4), for every $m \in \mathbb{N}$, we get

$$
\begin{aligned}
\xi\left(b_{m}\right) & =\sum_{\lambda \in F_{m}} \xi\left(b_{m, \lambda}\right)=\sum_{\lambda \in F_{m}} \lim _{n \rightarrow \infty} b_{m, \lambda}\left(h_{n}\right) \\
& =\lim _{n \rightarrow \infty} \sum_{\lambda \in F_{m}} b_{m, \lambda}\left(h_{n}\right)=\lim _{n \rightarrow \infty} b_{m}\left(h_{n}\right) .
\end{aligned}
$$

If the set $B_{\infty}$ is empty, we can take an arbitrary sequence $\left\{h_{n}\right\}_{n \in \mathbb{N}}$ such that $h_{n} \rightarrow+\infty$ as $n \rightarrow \infty$. 
Let $f \in \mathcal{S}_{0}(\mathbb{R})$. Then, by a smooth version of Urysohn's lemma (see, e.g., [1. Proposition 6.5]), there is a function $\psi \in C_{0}^{\infty}(\mathbb{R})$ such that $0 \leq \psi \leq 1$, $\operatorname{supp} \mathcal{F} f \subset \operatorname{supp} \psi$ and $\left.\psi\right|_{\operatorname{supp} \mathcal{F} f}=1$. Therefore, for all $n \in \mathbb{N}$,

$$
\begin{aligned}
e_{h_{n}} W^{0}(b) e_{h_{n}}^{-1} f-b(\xi) f & =W^{0}\left[b\left(\cdot+h_{n}\right)\right] f-\xi(b) f \\
& =\mathcal{F}^{-1}\left[b\left(\cdot+h_{n}\right)-\xi(b)\right] \psi \mathcal{F} f
\end{aligned}
$$

and

$$
\left\|\left(e_{h_{n}} W^{0}(b) e_{h_{n}}^{-1}-b(\xi)\right) f\right\|_{X(\mathbb{R})} \leq\left\|\left[b\left(\cdot+h_{n}\right)-\xi(b)\right] \psi\right\|_{\mathcal{M}_{X(\mathbb{R})}}\|f\|_{X(\mathbb{R})} .
$$

Since $\mathcal{M}_{X(\mathbb{R})}$ is translation-invariant and $\xi \in M_{\infty}\left(S O^{\diamond}\right)$ is a multiplicative linear functional on $S O_{X(\mathbb{R})}^{\diamond}$, we infer for all $m, n \in \mathbb{N}$ that

$$
\begin{aligned}
\left\|\left[b\left(\cdot+h_{n}\right)-\xi(b)\right] \psi\right\|_{\mathcal{M}_{X}(\mathbb{R}) \leq} & \left\|\left[b\left(\cdot+h_{n}\right)-b_{m}\left(\cdot+h_{n}\right)\right] \psi\right\|_{\mathcal{M}_{X(\mathbb{R})}} \\
& +\left\|\left[b_{m}\left(\cdot+h_{n}\right)-\xi\left(b_{m}\right)\right] \psi\right\|_{\mathcal{M}_{X(\mathbb{R})}} \\
& \left.+\| \xi\left(b_{m}\right)-\xi(b)\right] \psi \|_{\mathcal{M}_{X(\mathbb{R})}} \\
\leq & 2\left\|b-b_{m}\right\|_{\mathcal{M}_{X(\mathbb{R})}}\|\psi\|_{\mathcal{M}_{X(\mathbb{R})}} \\
& +\left\|\left[b_{m}\left(\cdot+h_{n}\right)-\xi\left(b_{m}\right)\right] \psi\right\|_{\mathcal{M}_{X(\mathbb{R})}}
\end{aligned}
$$

Fix $\varepsilon>0$. By Theorem 2.5, $\|\psi\|_{\mathcal{M}_{X(\mathbb{R})}}<\infty$. It follows from (5.2) that there exists a sufficiently large number $m \in \mathbb{N}$ (which we fix until the end of the proof) such that

$$
2\left\|b-b_{m}\right\|_{\mathcal{M}_{X(\mathbb{R})}}\|\psi\|_{\mathcal{M}_{X(\mathbb{R})}}<\varepsilon / 2 .
$$

Let

$$
\Lambda:=\left\{\begin{array}{lll}
\max _{\lambda \in F_{m} \backslash\{\infty\}}|\lambda| & \text { if } & F_{m} \backslash\{\infty\} \neq \emptyset, \\
0 & \text { if } & F_{m} \backslash\{\infty\}=\emptyset,
\end{array}\right.
$$

let $K:=\operatorname{supp} \psi$ and

$$
k:=\max \{-\inf K, \sup K\} \in[0, \infty) .
$$

For $x \in K$ and $n \in \mathbb{N}$, let $I_{n}(x)$ be the segment with the endpoints $h_{n}$ and $x+h_{n}$. Then $I_{n}(x) \subset\left[h_{n}-k, h_{n}+k\right]$. Since $h_{n} \rightarrow+\infty$ as $n \rightarrow \infty$, there exists $N_{1} \in \mathbb{N}$ such that for all $n>N_{1}$, one has

$$
I_{n}(x) \subset\left[h_{n}-k, h_{n}+k\right] \subset(\Lambda, \infty) .
$$

For all $n>N_{1}$, we have

$$
\begin{aligned}
\left\|\left[b_{m}\left(\cdot+h_{n}\right)-\xi\left(b_{m}\right)\right] \psi\right\|_{\mathcal{M}_{X}(\mathbb{R})} \leq & \left\|\left[b_{m}\left(\cdot+h_{n}\right)-b_{m}\left(h_{n}\right)\right] \psi\right\|_{\mathcal{M}_{X(\mathbb{R})}} \\
& +\left|b_{m}\left(h_{n}\right)-\xi\left(b_{m}\right)\right|\|\psi\|_{\mathcal{M}_{X(\mathbb{R})}}
\end{aligned}
$$

where the functions $\left[b_{m}\left(\cdot+h_{n}\right)-b_{m}\left(h_{n}\right)\right] \psi$ for $n>N_{1}$ belong to $S O_{\infty}^{3}$ because they are three times continuously differentiable functions of compact support.

By (5.5), there exists $N_{2} \in \mathbb{N}$ such that $N_{2} \geq N_{1}$ and for all $n>N_{2}$,

$$
\left|b_{m}\left(h_{n}\right)-\xi\left(b_{m}\right)\right|\|\psi\|_{\mathcal{M}_{X(\mathbb{R})}}<\varepsilon / 4 .
$$


On the other hand, since $\left[b_{m}\left(\cdot+h_{n}\right)-b_{m}\left(h_{n}\right)\right] \psi \in S O_{\infty}^{3}$ for all $n>N_{2}$, it follows from Theorem 2.7 that there exists a constant $c_{X}>0$ depending only on the space $X(\mathbb{R})$ such that for all $n>N_{2}$,

$$
\begin{aligned}
& \left\|\left[b_{m}\left(\cdot+h_{n}\right)-b_{m}\left(h_{n}\right)\right] \psi\right\|_{\mathcal{M}_{X(\mathbb{R})}} \\
& \quad \leq c_{X}\left\|\left[b_{m}\left(\cdot+h_{n}\right)-b_{m}\left(h_{n}\right)\right] \psi\right\|_{S O_{\infty}^{3}} \\
& \quad=c_{X} \sum_{j=0}^{3} \frac{1}{j !}\left\|D_{\infty}^{j}\left(\left[b_{m}\left(\cdot+h_{n}\right)-b_{m}\left(h_{n}\right)\right] \psi\right)\right\|_{L^{\infty}(\mathbb{R})} .
\end{aligned}
$$

For all $j \in\{0,1,2,3\}$, we have

$$
\begin{aligned}
D_{\infty}^{j}( & {\left.\left[b_{m}\left(\cdot+h_{n}\right)-b_{m}\left(h_{n}\right)\right] \psi\right) } \\
& =\sum_{\nu=0}^{j}\left(\begin{array}{l}
j \\
\nu
\end{array}\right)\left(D_{\infty}^{\nu}\left[b_{m}\left(\cdot+h_{n}\right)-b_{m}\left(h_{n}\right)\right]\right)\left(D_{\infty}^{j-\nu} \psi\right) .
\end{aligned}
$$

It follows from the mean value theorem that

$$
\begin{aligned}
\left\|\left[b_{m}\left(\cdot+h_{n}\right)-b_{m}\left(h_{n}\right)\right] \chi_{K}\right\|_{L^{\infty}(\mathbb{R})} & =\sup _{x \in K}\left|\int_{h_{n}}^{x+h_{n}} b_{m}^{\prime}(t) d t\right| \\
& =\sup _{x \in K}\left|\int_{h_{n}}^{x+h_{n}} t b_{m}^{\prime}(t) \frac{d t}{t}\right| \\
& \leq \sup _{x \in K} \int_{I_{n}(x)}\left|\left(D_{\infty} b_{m}\right)(t)\right| \frac{d t}{t} \\
& \leq \sup _{t \in\left[h_{n}-k, h_{n}+k\right]}\left|\left(D_{\infty} b_{m}\right)(t)\right| \int_{h_{n}-k}^{h_{n}+k} \frac{d t}{t} \\
& \leq \ln \frac{h_{n}+k}{h_{n}-k}\left\|D_{\infty} b_{m}\right\|_{L^{\infty}(\mathbb{R})} .
\end{aligned}
$$

It is easy to see that for $x \in K$,

$$
\begin{aligned}
& \left(D_{\infty}\left[b_{m}\left(\cdot+h_{n}\right)-b_{m}\left(h_{n}\right)\right]\right)(x)=\frac{x}{x+h_{n}}\left(D_{\infty} b_{m}\right)\left(x+h_{n}\right), \\
& \left(D_{\infty}^{2}\left[b_{m}\left(\cdot+h_{n}\right)-b_{m}\left(h_{n}\right)\right]\right)(x) \\
& \quad=\frac{x^{2}}{\left(x+h_{n}\right)^{2}}\left(D_{\infty}^{2} b_{m}\right)\left(x+h_{n}\right)+\frac{x h_{n}}{\left(x+h_{n}\right)^{2}}\left(D_{\infty} b_{m}\right)\left(x+h_{n}\right),
\end{aligned}
$$

and

$$
\begin{aligned}
\left(D_{\infty}^{3}\left[b_{m}\left(\cdot+h_{n}\right)-b_{m}\left(h_{n}\right)\right]\right)(x) & \\
= & \frac{x^{3}}{\left(x+h_{n}\right)^{3}}\left(D_{\infty}^{3} b_{m}\right)\left(x+h_{n}\right)+\frac{3 x^{2} h_{n}}{\left(x+h_{n}\right)^{3}}\left(D_{\infty}^{2} b_{m}\right)\left(x+h_{n}\right) \\
& \quad+\frac{x h_{n}^{2}-x^{2} h_{n}}{\left(x+h_{n}\right)^{3}}\left(D_{\infty} b_{m}\right)\left(x+h_{n}\right) .
\end{aligned}
$$


It follows from (5.14) -15.16 that for all $n>N_{2}$,

$$
\begin{aligned}
& \left\|\left(D_{\infty}\left[b_{m}\left(\cdot+h_{n}\right)-b_{m}\left(h_{n}\right)\right]\right) \chi_{K}\right\|_{L^{\infty}(\mathbb{R})} \leq \frac{k}{h_{n}-k}\left\|D_{\infty} b_{m}\right\|_{L^{\infty}(\mathbb{R})}, \\
& \left\|\left(D_{\infty}^{2}\left[b_{m}\left(\cdot+h_{n}\right)-b_{m}\left(h_{n}\right)\right]\right) \chi_{K}\right\|_{L^{\infty}(\mathbb{R})} \\
& \quad \leq \frac{k^{2}}{\left(h_{n}-k\right)^{2}}\left\|D_{\infty}^{2} b_{m}\right\|_{L^{\infty}(\mathbb{R})}+\frac{k h_{n}}{\left(h_{n}-k\right)^{2}}\left\|D_{\infty} b_{m}\right\|_{L^{\infty}(\mathbb{R})},
\end{aligned}
$$

and

$$
\begin{aligned}
& \left\|\left(D_{\infty}^{3}\left[b_{m}\left(\cdot+h_{n}\right)-b_{m}\left(h_{n}\right)\right]\right) \chi_{K}\right\|_{L^{\infty}(\mathbb{R})} \\
& \leq \frac{k^{3}}{\left(h_{n}-k\right)^{3}}\left\|D_{\infty}^{3} b_{m}\right\|_{L^{\infty}(\mathbb{R})}+\frac{3 k^{2} h_{n}}{\left(h_{n}-k\right)^{3}}\left\|D_{\infty}^{2} b_{m}\right\|_{L^{\infty}(\mathbb{R})} \\
& \quad+\frac{k h_{n}^{2}+k^{2} h_{n}}{\left(h_{n}-k\right)^{3}}\left\|D_{\infty} b_{m}\right\|_{L^{\infty}(\mathbb{R})}
\end{aligned}
$$

Since

$$
\max _{j \in\{0,1,2,3\}}\left\|D_{\infty}^{j} \psi\right\|_{L^{\infty}(\mathbb{R})}<\infty
$$

it follows from (5.12) - (5.13) and (5.17)

$$
\lim _{n \rightarrow \infty}\left\|D_{\infty}^{j}\left(\left[b_{m}\left(\cdot+h_{n}\right)-b_{m}\left(h_{n}\right)\right] \psi\right)\right\|_{L^{\infty}(\mathbb{R})}=0
$$

We deduce from (5.11) and (5.20) that there exists $N_{3} \in \mathbb{N}$ such that $N_{3} \geq N_{2}$ and for all $n>N_{3}$,

$$
\left\|\left[b_{m}\left(\cdot+h_{n}\right)-b_{m}\left(h_{n}\right)\right] \psi\right\|_{\mathcal{M}_{X(\mathbb{R})}}<\varepsilon / 4 .
$$

Combining (5.7)- (5.10) and (5.21), we see that for every $f \in \mathcal{S}_{0}(\mathbb{R})$ and every $\varepsilon>0$ there exists $N_{3} \in \mathbb{N}$ such that for all $n>N_{3}$,

$$
\left\|\left(e_{h_{n}} W^{0}(b) e_{h_{n}}^{-1}-b(\xi)\right) f\right\|_{X(\mathbb{R})}<\varepsilon\|f\|_{X(\mathbb{R})},
$$

whence for all $f \in \mathcal{S}_{0}(\mathbb{R})$,

$$
\lim _{n \rightarrow \infty}\left\|\left(e_{h_{n}} W^{0}(b) e_{h_{n}}^{-1} I-b(\xi) I\right) f\right\|_{X(\mathbb{R})}=0 .
$$

Since $\mathcal{S}_{0}(\mathbb{R})$ is dense in $X(\mathbb{R})$ (see Theorem $[2.2$ ), this equality immediately implies (5.1) in view of [28, Lemma 1.4.1(ii)], which completes the proof.

\subsection{Proof of Theorem 1.1}

Since the function $e_{0} \equiv 1$ belongs to $\Phi$ and $\Psi_{S O_{X(\mathbb{R})}^{\diamond}}$, we see that the set of all constant functions is contained in $\Phi$ and in $S O_{X(\mathbb{R})}^{\diamond}$. Therefore

$$
\mathcal{M O}^{\pi}(\mathbb{C}) \subset \mathcal{M O}^{\pi}(\Phi) \cap \mathcal{C O}^{\pi}\left(S O_{X(\mathbb{R})}^{\diamond}\right)
$$

Let $A^{\pi} \in \mathcal{M O}^{\pi}(\Phi) \cap \mathcal{C} \mathcal{O}^{\pi}\left(S O_{X(\mathbb{R})}^{\diamond}\right)$. Then $A^{\pi}=[a I]^{\pi}=\left[W^{0}(b)\right]^{\pi}$, where $a \in \Phi$ and $b \in S O_{X(\mathbb{R})}^{\diamond}$. Therefore, there is an operator $K \in \mathcal{K}(X(\mathbb{R}))$ such that

$$
a I=W^{0}(b)+K .
$$


By Theorem 5.2, for every $\xi \in M_{\infty}\left(S O^{\diamond}\right)$ there exists a sequence $\left\{h_{n}\right\}_{n \in \mathbb{N}}$ of positive numbers such that $h_{n} \rightarrow+\infty$ as $n \rightarrow \infty$ and

$$
\text { s- } \lim _{n \rightarrow \infty} e_{h_{n}} W^{0}(b) e_{h_{n}}^{-1} I=b(\xi) I .
$$

Equalities (5.23)-(5.24) and Lemma 5.1 imply that

$$
a I=\mathrm{s}_{n \rightarrow \infty} \lim _{h_{n}}(a I) e_{h_{n}}^{-1} I=\mathrm{s}_{n \rightarrow \infty} \lim _{h_{n}}\left(W^{0}(b)+K\right) e_{h_{n}}^{-1} I=b(\xi) I .
$$

Hence $[a I]^{\pi}=[b(\xi) I]^{\pi} \in \mathcal{M O}^{\pi}(\mathbb{C})$ and

$$
\mathcal{M O}^{\pi}(\Phi) \cap \mathcal{C O}^{\pi}\left(S O_{X(\mathbb{R})}^{\diamond}\right) \subset \mathcal{M O}^{\pi}(\mathbb{C}) .
$$

Combining (5.22) and (5.25), we arrive at (1.4).

\section{Acknowledgment}

We would like to thank the anonymous referee for pointing out a gap in the original version of the paper. To fill in this gap, we strengthened the hypotheses in the main result.

\section{References}

[1] J. Anderson, Extensions, restrictions, and representations of states on $C^{*}$ algebras, Trans. Amer. Math. Soc. 249 (1979), 303-329.

[2] M. A. Bastos, A. Bravo, and Yu. I. Karlovich, Convolution type operators with symbols generated by slowly oscillating and piecewise continuous matrix functions, Oper. Theory Adv. Appl. 147 (2004), 151-174.

[3] M. A. Bastos, C. A. Fernandes, and Yu. I. Karlovich, $C^{*}$-algebras of integral operators with piecewise slowly oscillating coefficients and shifts acting freely, Integr. Equ. Oper. Theory 55 (2006), 19-67.

[4] C. Bennett and R. Sharpley, Interpolation of Operators, Academic Press, Boston, 1988.

[5] A. Böttcher, Yu. I. Karlovich, and I. M. Spitkovsky, Convolution Operators and Factorization of Almost Periodic Matrix Functions, Birkhäuser, Basel, 2002.

[6] D. Cruz-Uribe and A. Fiorenza, Variable Lebesgue Spaces, Birkhäuser/Springer, New York, 2013.

[7] R. V. Duduchava, Integral Equations with Fixed Singularities, Teubner, Leipzig, 1979.

[8] C. A. Fernandes, A. Yu. Karlovich, and Yu. I. Karlovich, Noncompactness of Fourier convolution operators on Banach function spaces, Ann. Funct. Anal. AFA 10 (2019), 553-561.

[9] C. A. Fernandes, A. Yu. Karlovich, and Yu. I. Karlovich, Algebra of convolution type operators with continuous data on Banach function spaces, Banach Center Publications 119 (2019), 157-171.

[10] C. A. Fernandes, A. Yu. Karlovich, and Yu. I. Karlovich, Fourier convolution operators with symbols equivalent to zero at infinity on Banach function spaces, Proceedings of ISAAC 2019, to appear. Preprint is available at arXiv:1909.13538 [math.FA]. 
[11] G. B. Folland, A Guide to Advanced Real Analysis, The Mathematical Association of America, Washington, DC, 2009.

[12] L. Grafakos, Classical Fourier Analysis, 3rd ed., Springer, New York, 2014.

[13] I. Gohberg and N. Krupnik, One-Dimensional Linear Singular Integral Equations. Vol. II, Birkhäuser, Basel, 1992.

[14] H. Hudzik, R. Kumar, and R. Kumar, Matrix multiplication operators on Banach function spaces, Proc. Indian Acad. Sci., Math. Sci. 116 (2006), 7181.

[15] R. V. Kadison and J. R Ringrose, Fundamentals of the Theory of Operator Algebras. Vol. I: Elementary Theory, 2nd ed. American Mathematical Society, Providence, RI, 1997.

[16] E. Kaniuth, A Course in Commutative Banach Algebras, Springer, New York, 2009.

[17] A. Yu. Karlovich, Maximally modulated singular integral operators and their applications to pseudodifferential operators on Banach function spaces, Contemp. Math. 645 (2015), 165-178.

[18] A. Yu. Karlovich, Banach algebra of the Fourier multipliers on weighted Banach function spaces, Concr. Oper. 2 (2015), 27-36.

[19] A. Yu. Karlovich, Commutators of convolution type operators on some Banach function spaces, Ann. Funct. Anal. AFA 6 (2015), 191-205.

[20] A. Yu. Karlovich, The Stechkin inequality for Fourier multipliers on variable Lebesgue spaces, Math. Inequal. Appl. 18 (2015), 1473-1481.

[21] A. Karlovich and E. Shargorodsky, When does the norm of a Fourier multiplier dominate its $L^{\infty}$ norm? Proc. London Math. Soc. 118 (2019), 901-941.

[22] A. Yu. Karlovich and I. M. Spitkovsky, The Cauchy singular integral operator on weighted variable Lebesgue spaces, Oper. Theory Adv. Appl. 236 (2014), 275-291.

[23] Yu. I. Karlovich, Algebras of convolution-type operators with piecewise slowly oscillating data on weighted Lebesgue spaces, Mediterr. J. Math. 14 (2017), paper no. 182, 20 p.

[24] Yu. I. Karlovich and I. Loreto Hernández, Algebras of convolution type operators with piecewise slowly oscillating data. I: Local and structural study, Integr. Equ. Oper. Theory 74 (2012), 377-415.

[25] Yu. I. Karlovich and I. Loreto Hernández, On convolution type operators with piecewise slowly oscillating data, Oper. Theory Adv. Appl. 228 (2013), 185207.

[26] V. Rabinovich, S. Roch, and B. Silbermann, Limit Operators and Their Applications in Operator Theory, Birkhäuser, Basel, 2004.

[27] M. Reed and B. Simon, Methods of Modern Mathematical Physics. I: Functional Analysis, Academic Press, New York, 1980.

[28] S. Roch, P. A. Santos, and B. Silbermann, Non-Commutative Gelfand Theories. A Tool-kit for Operator Theorists and Numerical Analysts, Springer, Berlin, 2011.

[29] I. B. Simonenko, Local Method in the Theory of Shift Invariant Operators and Their Envelopes, Rostov Univ. Press, Rostov on Don, 2007 (in Russian). 
[30] I. B. Simonenko and Chin Ngok Min, Local Method in the Theory of OneDimensional Singular Integral Equations with Piecewise Continuous Coeffcients. Noetherity, Rostov Univ. Press, Rostov on Don, 1986 (in Russian).

C. A. Fernandes

Centro de Matemática e Aplicações,

Departamento de Matemática,

Faculdade de Ciências e Tecnologia,

Universidade Nova de Lisboa,

Quinta da Torre,

2829-516 Caparica, Portugal

e-mail: caf@fct.unl.pt

A. Yu. Karlovich

Centro de Matemática e Aplicações,

Departamento de Matemática,

Faculdade de Ciências e Tecnologia,

Universidade Nova de Lisboa,

Quinta da Torre,

2829-516 Caparica, Portugal

e-mail: oyk@fct.unl.pt

Yu. I. Karlovich

Centro de Investigación en Ciencias,

Instituto de Investigación en Ciencias Básicas y Aplicadas,

Universidad Autónoma del Estado de Morelos,

Av. Universidad 1001, Col. Chamilpa,

C.P. 62209 Cuernavaca, Morelos, México

e-mail: karlovich@uaem.mx 